\title{
Corela
}

Cognition, représentation, langage

HS-17 | 2015

Regards sur l'interdisciplinarité en science du langage

\section{De la didactique des sciences à la didactique de} l'orthographe

Interdisciplinarité et perspectives d'exploitation de protocoles

\section{Thierry Geoffre}

\section{OpenEdition}

\section{Journals}

Édition électronique

URL : http://journals.openedition.org/corela/3731

DOI : $10.4000 /$ corela.3731

ISSN : 1638-573X

Éditeur

Cercle linguistique du Centre et de l'Ouest - CerLICO

Référence électronique

Thierry Geoffre, « De la didactique des sciences à la didactique de l'orthographe », Corela [En ligne], HS-17 | 2015, mis en ligne le 30 mai 2015, consulté le 30 avril 2019. URL : http://

journals.openedition.org/corela/3731; DOI : 10.4000/corela.3731

Ce document a été généré automatiquement le 30 avril 2019

\section{c) (i) (2)}

Corela - cognition, représentation, langage est mis à disposition selon les termes de la licence Creative Commons Attribution - Pas d'Utilisation Commerciale - Partage dans les Mêmes Conditions 4.0 International. 


\section{De la didactique des sciences à la didactique de l'orthographe}

Interdisciplinarité et perspectives d'exploitation de protocoles

Thierry Geoffre

\section{Introduction}

1 Cet article propose une réflexion sur des dispositifs didactiques permettant d'aborder l'étude de la langue, et plus particulièrement l'orthographe grammaticale, avec des élèves de cycle 3 de l'école primaire (enfants âgés de 8 à 11 ans) dans une démarche de dépassement des représentations de l'apprenant et de transformation de son système orthographique.

2 La didactique du français langue maternelle, en tant que discipline, s'est développée plus tardivement que les didactiques des mathématiques et des sciences et s'est rapidement nourrie de certains des concepts élaborés par ces autres champs de réflexion. La transposition didactique proposée par Yves Chevallard en 1985 ou le contrat didactique de Guy Brousseau en 1989, par exemple, originellement proposés par des didacticiens des mathématiques, sont rapidement intégrés au discours de didactique du français (Halté, 1992). Claudine Garcia- Debanc (1990) a dressé un intéressant état des lieux des convergences entre didactique du français et didactique des disciplines scientifiques. Elle y indique que la didactique du français a également ses propres spécificités, notamment parce qu'elle apparaît comme une " confédération de didactiques " se rapportant à des domaines spécifiques (lecture, production écrite, orthographe, vocabulaire, grammaire). Une autre spécificité est pointée : le caractère autonymique de la langue. Le français utilise le langage naturel comme outil de connaissance et d'enseignement mais également comme objet d'étude. On voit les implications de cette autonymie pour le jeune apprenant, notamment dans les risques de confusions langagières ou les difficultés à détacher raisonnement grammatical et dimension sémantique de la langue. Alors, peuton étudier la langue comme un objet scientifique, mis à distance ? En ce cas, peut-on 
s'inspirer de protocoles issus des didactiques des champs scientifiques pour aider l'élève à appréhender la langue et modifier son système de représentations?

Une première réflexion laisse à penser que la norme en sciences du langage ne pourra pas être le «vrai» comme dans les enseignements scientifiques (celui du résultat d'expérience, par exemple) mais sera la rationalité du discours qui «permet d'évoquer des faits et les expliquer selon les normes de la grammaticalité sans pour autant atteindre les véritables fondements conceptuels » (Sarremajane, 2001). De fait, les savoirs enseignés en français ne sont pas seulement savants mais engagent aussi une part que l'on qualifiera de « culturelle ».

Concernant l'apprenant, Michel Dabène (1987) a montré que trois types de savoirs interviennent dans les apprentissages en français :

- des savoirs appris à l'école ;

- des savoirs acquis dans la sphère sociale par les interactions avec la famille, les pairs... ;

- des savoirs hérités liés aux représentations d'appartenance socio-culturelle.

Il apparait donc que, en didactique du français, chaque sommet du triangle didactique (enseignant - savoir - apprenant) porte une «marque sociale » faite de représentations, d'expériences, de contraintes. Et nous voyons au quotidien à quel point les représentations sur la langue, son enseignement, et ce qui doit être enseigné, sont saillantes dans la société.

Une réponse possible à la question initiale d'une étude de la langue comme objet scientifique serait donc la mise à distance de cette langue quotidienne, de ces spécificités de l'enseignement du français, pour progresser vers une étude scientifique. On peut envisager de l'obtenir par le recours à une activité métalinguistique, définie comme une activité de réflexion sur la langue et son utilisation. Cette réflexion sur la langue exige d'avoir des mots pour nommer ce qu'on observe et l'adoption d'un métalangage commun à la classe est nécessaire. C'est lui qui devient alors le langage technique, le langage d'analyse. Nous soutiendrons, à la suite de Suzanne Chartrand (1995), la nécessité de réévaluer et de redéfinir le métalangage grammatical à partir de critères de classification rigoureux et cohérents puisqu'il est lui-même, parfois, utilisé hors contexte ou fortement marqué socialement1. L'auteure précise qu'il vaut mieux " adopter un métalangage limité, mais dont la signification est, pour les élèves, la moins ambigüe et la moins polysémique possible ». On voit dès lors apparaitre de nouveaux enjeux pour l'enseignant qui doit conduire les élèves à parler sur leur langue avec un nouveau vocabulaire. Un vocabulaire qu'ils vont devoir comprendre et apprendre. Un vocabulaire qui permettra aussi de se détacher de la valeur sémantique de l'énoncé pour se concentrer sur la syntaxe.

\section{L'étude de la langue à l'école primaire}

Parmi les domaines de la didactique du français langue maternelle, nous considérons que celui de l'orthographe grammaticale est potentiellement le plus proche des sciences : de fortes théories linguistiques existent comme savoirs référents, leurs transpositions didactiques tentent d'établir des régularités et des justifications des faits de langue. L'ensemble forme un système sur lequel il est possible d'émettre des hypothèses et de les valider. Les outils sont alors le métalangage et les méthodes d'analyse orthographique et grammaticale des chaines syntagmatiques. Des recherches récentes (Cogis, 2005 ; Geoffre 
\& Brissaud, 2012) définissent des procédures cognitives mises en place dans l'apprentissage et la gestion de l'orthographe grammaticale. Ces procédures reposent sur une analyse morphosémantique et/ou morphosyntaxique de la phrase.

Notre réflexion pose que chaque élève construit, à partir de ses savoirs appris, acquis et hérités, son propre système orthographique, c'est-à-dire un système de représentations de la langue et de son fonctionnement qui va servir de système de justification de ses choix orthographiques (lorsque l'élève est apte à le faire). Dès lors, les procédures cognitives utilisées par l'apprenant forment un système explicatif avec ses limites, des limites qu'il faudra confronter à la réalité de « l'expérience " pour les faire évoluer, tout comme en didactique des sciences expérimentales.

Si l'on considère les propositions d'Odile Guyon (1997) de " réfléchir aux conditions favorables à rendre le concept d'accord sujet-verbe opérationnel et aux activités permettant à l'élève de construire une représentation la plus juste possible du système orthographique " ou encore de "caractériser ce qu'est un accord de proximité et ses limites, ce qui devient obstacle et conduit à l'erreur » (Guyon, 1997 : 38), le parallèle avec les objectifs des didactiques des sciences semble possible sur au moins deux points :

- le travail sur l'erreur comme appui des apprentissages ;

- le travail sur l'objet d'enseignement, effectivement perçu comme un système, mais sur lequel l'apprenant a des représentations qui peuvent devenir des obstacles.

Il pourrait donc exister une similitude épistémologique entre didactique de l'orthographe grammaticale et didactique des disciplines scientifiques. Il serait alors envisageable de problématiser l'étude de la langue et de confronter l'élève à ses démarches ou représentations en orthographe grammaticale pour les faire évoluer.

\section{Phrase dictée, phrase donnée, phrase dictée/ donnée}

11 Dans le cadre du suivi longitudinal pendant trois ans d'une classe d'élèves de cycle 3 (classe multi-niveaux: CE2 - CM1 - CM2), nous avons mené plusieurs séquences expérimentales. La première (février - avril 2009) exploitait les propositions de deux activités courtes et ritualisées : la phrase dictée du jour et la phrase donnée du jour (Cogis, 2005). Dans la phrase dictée du jour, l'enseignant dicte une phrase que chaque élève écrit individuellement. Le travail est ensuite repris en groupe avant de conduire à une synthèse collective. Le temps passé en groupe doit favoriser le conflit socio-cognitif et la négociation. La phrase donnée du jour propose, elle, un cheminement cognitif différent et complémentaire, en quelque sorte inverse : l'enseignant donne une phrase et demande aux élèves d'en justifier l'orthographe, sachant qu'elle est correcte. De la même manière, le temps individuel est suivi d'une phase de groupe puis d'une synthèse collective.

Deux éléments de réflexion ont émergé à la suite de la première séquence expérimentale :

- la complémentarité des deux dispositifs nous a rapidement conduit à les alterner d'une séance à l'autre plutôt que de mener, à la suite, une série de phrases dictées uniquement ou de phrases données uniquement ;

- la phrase donnée du jour, dans ce déroulement alternant temps individuels et collectifs, nous a semblé se rapprocher d'un cheminement de situation-problème, où l'observation d'une situation initiale sert de levier pour " trancher " entre des systèmes explicatifs 
contradictoires, co-présents dans la même classe et proposés par les élèves, à la manière de la première stratégie de dépassement des représentations proposée par le groupe ASTER, où la résolution du problème passe par l'élaboration d'une situation qui permettra de garder ou non un système explicatif (1985). Est alors recherchée l'émergence de conflits socio-cognitifs qui visent le dépassement de représentations ou de raisonnements non opérants, à condition que les élèves aient la possibilité de confronter leurs propositions. C'est l'analyse grammaticale globale de la phrase, en synthèse collective, qui validera un système explicatif ou pas. Il y a bien un objet d'étude scientifique : la langue. Et il y a bien un système de référence : la théorie grammaticale sur laquelle s'appuie l'enseignement de la classe. Nous transformons ainsi le schéma initial proposé par le groupe ASTER selon le schéma du tableau 1.

La différence de dispositif et de sollicitation cognitive entre la phrase dictée du jour et la phrase donnée du jour nous semble importante puisque, si l'on cherche un conflit sociocognitif dans les deux cas, il est activé soit par la confrontation entre des choix orthographiques individuels et leurs justifications (phrase dictée) soit par la confrontation entre systèmes explicatifs individuels cherchant la justification de la phrase observée. Cependant, nous constatons que, même en alternant les dispositifs, l'élève n'est jamais confronté individuellement à une limite de son propre système explicatif. La remise en question vient des autres apprenants, elle n'est pas interne. Notre réflexion s'est donc tournée vers la recherche de modalités susceptibles d'activer un conflit cognitif dès la phase individuelle, avant d'engager l'éventuel conflit socio-cognitif de la phase de groupe.

Tableau 1. - Dépassement de représentations par activation de conflits socio-cognitifs : phrase dictée du jour

Tableau I. - Dépassement de représentations par activation de conflits sociocognitifs

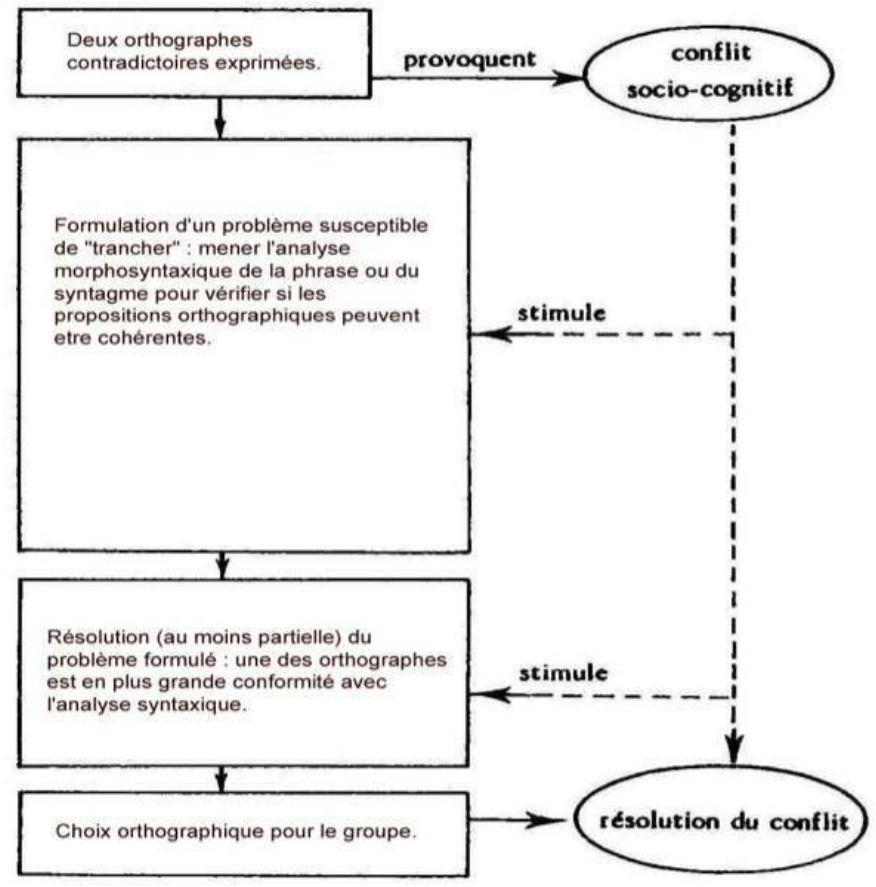

La deuxième stratégie proposée par le groupe ASTER, dite de " dépassement de représentations par recherches de limites » (1985), apporte une opportunité intéressante 
pour y parvenir. Cette fois-ci, dans le cadre de la didactique des sciences, il s'agit de confronter la prévision d'une représentation ou d'un système explicatif avec l'observation. Si la prévision ne correspond pas à l'observation, il y a déstabilisation de la représentation et activation du conflit cognitif. Nous avons alors cherché un déroulement qui permettrait de confronter directement l'élève à une incompatibilité entre sa représentation de la morphographie et l'observable afin de déstabiliser son système orthographique. Ainsi, il s'agirait de mettre très rapidement en relation la phrase écrite par l'élève sous la dictée (sa "prévision» de l'orthographe du texte) avec la phrase donnée par l'enseignant (qui joue le rôle d'observable). Suite à l'écriture de la phrase dictée, la phrase donnée serait présentée et il serait demandé à l'élève de relever les écarts éventuels entre les deux écritures (sur des items ciblés) et de justifier l'écriture de référence. L'élève entrerait alors dans un conflit cognitif individuel, "personnel », lui demandant de justifier une orthographe qui ne correspond pas à celle qu'il vient de produire et pour laquelle il avait un système explicatif dont il constate les limites.

15 Sur la base du deuxième schéma du groupe ASTER, nous proposons le dispositif suivant, que nous avons nommé phrase dictée/donnée (tableau 2) :

Tableau 2. - Dépassement de représentations par recherches de limites : phrase dictée/donnée
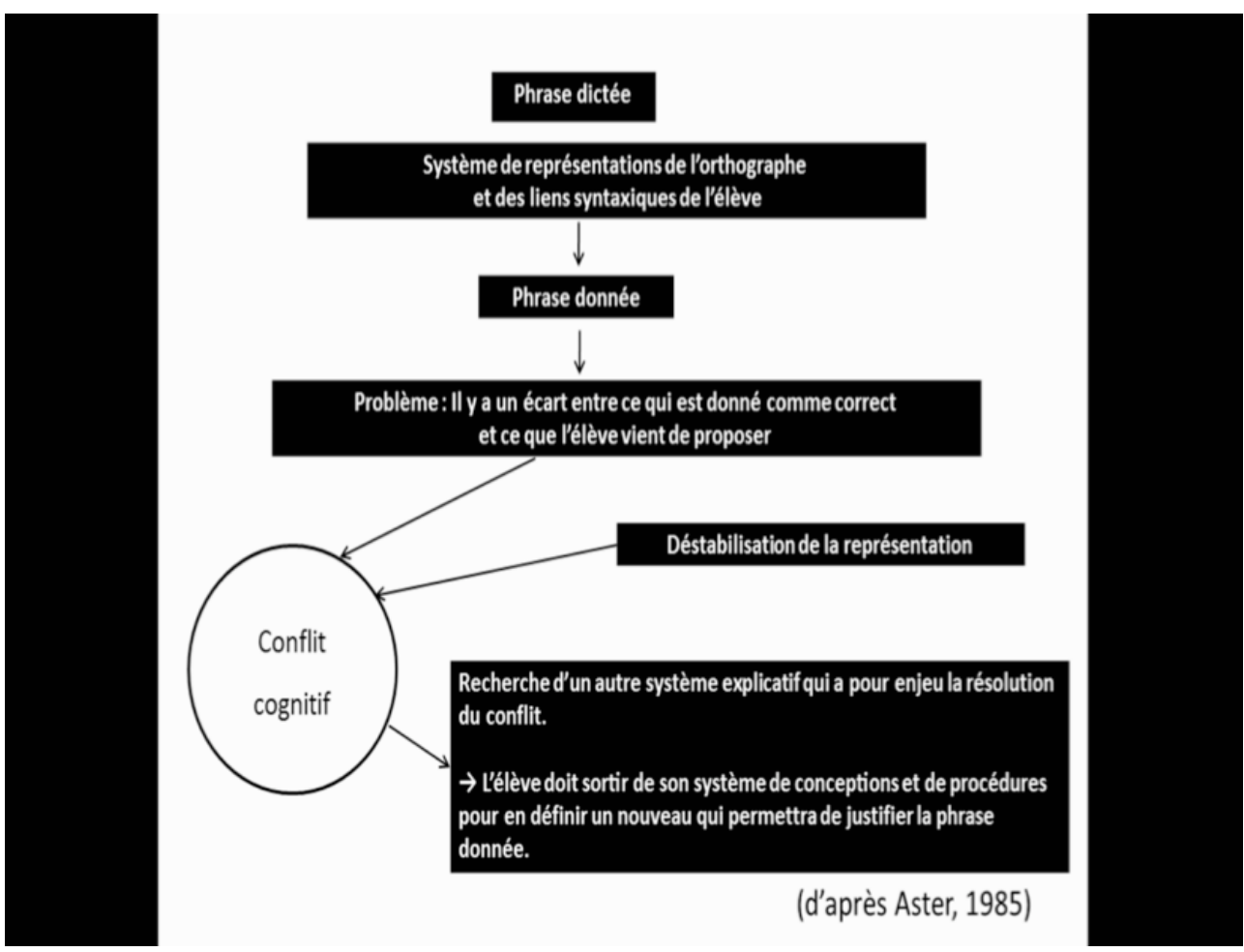

La deuxième séquence expérimentale, menée de février à avril 2010 (2 $2^{\text {ème }}$ année du suivi longitudinal), dans la même classe de cycle 3 (triple niveau CE2 - CM1 - CM2), s'est appuyée sur ce nouveau dispositif pour le développer et vérifier son efficience. L'objectif pédagogique de cette séquence portait sur un approfondissement des notions abordées lors de la première séquence: accords en genre et nombre dans le groupe nominal et accord sujet-verbe. Nous avons fait le choix d'engager la classe dans un travail pointu d'observation de chaines syntagmatiques contenant des noms massifs ou collectifs2 ainsi que sur des phrases où le verbe conjugué est éloigné du sujet ou antéposé. Dans ces situations, le traitement des chaines syntagmatiques par des procédures 
morphosémantiques ou par accord de proximité (majoritaires dans le travail des élèves en début de séquence, plus particulièrement pour les CE2) conduit à des choix orthographiques erronés ou ne peut pas expliquer l'orthographe constatée. Il y a donc nécessité d'accéder à des procédures morphosyntaxiques pour lever l'obstacle. La séquence visait donc la déstabilisation de ces procédures initiales pour leur substituer de nouvelles procédures, plus efficientes dans ces contextes syntaxiques.

La troisième séquence expérimentale menée dans la classe, de février à avril 2010 (3 3 ème année du suivi), s'est également appuyée sur le dispositif des phrases dictées/données et visait la discrimination des formes verbales homophones en /E/ (imparfait, infinitif en -er , participe passé en -é) avant d'engager les procédures d'accord. Nous avons défini un protocole de la phrase dictée/donnée, mené en deux temps distincts pour favoriser une reprise en différé de l'activité de groupe avant la phase collective. Le protocole est présenté dans le tableau 3. On notera le maintien d'une phase individuelle (conflit cognitif) puis d'une phase de groupe (conflit socio-cognitif) avant la synthèse collective de validation.

Tableau 3. - Protocole de la phrase dictée/donnée

\begin{tabular}{|c|c|c|}
\hline \multirow{4}{*}{$\begin{array}{l}\text { temps } \\
1\end{array}$} & \multirow{3}{*}{$\begin{array}{l}\text { phase } \\
\text { individuelle }\end{array}$} & phrase dictée à la classe \\
\hline & & phrase donnée crite au tableau \\
\hline & & $\begin{array}{l}\text { justification de la phrase donnée (nécessité de justifier une } \\
\text { orthographe qui n'est pas celle qui vient d'être produite par l'élève) }\end{array}$ \\
\hline & $\begin{array}{l}\text { phase de } \\
\text { groupe }\end{array}$ & $\begin{array}{l}\text { chaque groupe, sur la base des productions individuelles, doit produire } \\
\text { une nouvelle série de justifications de la phrase donnée et l'écrire sur } \\
\text { un panneau qui est affiché au tableau }\end{array}$ \\
\hline \multirow{3}{*}{$\begin{array}{l}\text { temps } \\
2\end{array}$} & $\begin{array}{l}\text { phase de } \\
\text { groupe }\end{array}$ & $\begin{array}{l}\text { (reprise en différé) } \\
\text { modifications éventuelles avant présentation }\end{array}$ \\
\hline & \multirow{2}{*}{$\begin{array}{l}\text { synthèse } \\
\text { collective }\end{array}$} & $\begin{array}{l}\text { chaque groupe présente son travail et ses arguments au reste de la } \\
\text { classe }\end{array}$ \\
\hline & & phase de validation \\
\hline
\end{tabular}

\section{Résultats et discussion}

Les passations d'un même test à trois moments-clés (dictée et questionnaire métagraphique avant la séquence, après la séquence puis deux mois plus tard) lors des deuxième et troisième séquences expérimentales nous servent à mesurer l'évolution des productions des élèves. Les items observés relèvent de l'accord de substantifs au pluriel, d'adjectifs au pluriel et/ou au féminin, et, selon la séquence :

- de verbes au pluriel ou bien de verbes au singulier avec sujet massif ou collectif (deuxième séquence); 
- de verbes conjugués à l'imparfait, de participes passés en -é employés dans des passés composés ou comme adjectifs, d'infinitifs en -er (troisième séquence).

Nous présentons ici quelques résultats quantitatifs issus de la deuxième séquence expérimentale qui exploitait la première mise en pratique du dispositif. Lors de cette séquence, les résultats généraux (tableau 4) ne font pas ressortir de progression nette sur les items difficiles d'accord verbal ou nominal liés aux noms collectifs et massifs. En revanche, l'évolution est plus marquée en ce qui concerne le pluriel verbal, le verbe avec sujet postposé ainsi que l'accord de l'adjectif en genre et nombre. C'est-à-dire que ce sont des compétences prioritaires de la maitrise orthographique au cycle 3 de l'école primaire qui semblent améliorées. Une interprétation renforcée par l'analyse qualitative des réponses produites au questionnaire métagraphique qui traduit une nette amélioration de la pertinence des réponses et de l'entrée dans une posture métalinguistique accompagnée d'une meilleure maitrise du métalangage.

Tableau 4. - Évolution des réussites pour la classe (séquence 2)

\begin{tabular}{|l|l|l|l|}
\hline classe (N=23 élèves) & Pré-test & Post-test 1 & Post-test 2 \\
\hline verbes (pluriel) & $46 \%$ & $61 \%$ & $54 \%$ \\
\hline verbe avec S postposé & $39 \%$ & $39 \%$ & $57 \%$ \\
\hline noms (pluriel) & $82 \%$ & $86 \%$ & $79 \%$ \\
\hline noms collectifs / massifs & $38 \%$ & $42 \%$ & $43 \%$ \\
\hline adjectifs (pluriel) & $66 \%$ & $76 \%$ & $67 \%$ \\
\hline adjectifs (féminin) & $35 \%$ & $47 \%$ & $52 \%$ \\
\hline réussite globale & $64 \%$ & $68 \%$ & $64 \%$ \\
\hline
\end{tabular}

L'analyse quantitative par niveau scolaire montre une évolution comparable et progressive des réussites. La progression des CE2 est remarquable si l'on considère certains items et leur difficulté relative à ce niveau : verbe avec sujet postposé, accord en nombre du verbe et de l'adjectif (tableau 5).

Tableau 5. - Évolution des réussites pour les élèves de CE2 (séquence 2)

\begin{tabular}{|l|l|l|l|}
\hline CE2 (N=8) & Pré-test & Post-test 1 & Post-test 2 \\
\hline verbes (pluriel) & $25 \%$ & $46 \%$ & $38 \%$ \\
\hline verbe avec S postposé & $50 \%$ & $63 \%$ & $88 \%$ \\
\hline noms (pluriel) & $74 \%$ & $83 \%$ & $80 \%$ \\
\hline noms collectifs / massifs & $29 \%$ & $29 \%$ & $46 \%$ \\
\hline
\end{tabular}




\begin{tabular}{|l|l|l|l|}
\hline adjectifs (pluriel) & $53 \%$ & $80 \%$ & $63 \%$ \\
\hline adjectifs (féminin) & $56 \%$ & $50 \%$ & $56 \%$ \\
\hline réussite globale & $56 \%$ & $64 \%$ & $63 \%$ \\
\hline
\end{tabular}

21 De même, les élèves de CM1 améliorent sensiblement leurs réussites de l'accord verbal et adjectival (tableau 6) alors que les CM2 progressent sur des notions plus complexes : noms collectifs et massifs, accord en genre de l'adjectif (tableau 7).

Tableau 6. - Évolution des réussites pour les élèves de CM1 (séquence 2)

\begin{tabular}{|l|l|l|l|}
\hline CM1 (N=8) & Pré-test & Post-test 1 & Post-test 2 \\
\hline verbes (pluriel) & $42 \%$ & $71 \%$ & $67 \%$ \\
\hline verbe avec S postposé & $13 \%$ & $13 \%$ & $38 \%$ \\
\hline noms (pluriel) & $85 \%$ & $80 \%$ & $84 \%$ \\
\hline noms collectifs / massifs & $38 \%$ & $38 \%$ & $42 \%$ \\
\hline adjectifs (pluriel) & $70 \%$ & $68 \%$ & $78 \%$ \\
\hline adjectifs (féminin) & $38 \%$ & $44 \%$ & $50 \%$ \\
\hline réussite globale & $63 \%$ & $62 \%$ & $68 \%$ \\
\hline
\end{tabular}

Tableau 7. - Évolution des réussites pour les élèves de CM2 (séquence 2)

\begin{tabular}{|l|l|l|l|}
\hline CM2 (N=7) & Pré-test & Post-test 1 & Post-test 2 \\
\hline verbes (pluriel) & $76 \%$ & $67 \%$ & $71 \%$ \\
\hline verbe avec S postposé & $57 \%$ & $43 \%$ & $57 \%$ \\
\hline noms (pluriel) & $87 \%$ & $96 \%$ & $91 \%$ \\
\hline noms collectifs / massifs & $48 \%$ & $62 \%$ & $52 \%$ \\
\hline adjectifs (pluriel) & $77 \%$ & $80 \%$ & $80 \%$ \\
\hline adjectifs (féminin) & $57 \%$ & $71 \%$ & $64 \%$ \\
\hline réussite globale & $74 \%$ & $78 \%$ & $76 \%$ \\
\hline
\end{tabular}

22 On observe donc un progrès global de la classe, progressif selon le niveau scolaire concerné, pour des situations impliquant un calcul syntaxique afin d'obtenir une écriture normée. Pour certains items, et en fonction du groupe considéré, on note des régressions 
apparentes. L'analyse détaillée, niveau par niveau, puis élève par élève, montre en fait une évolution ou une transformation des productions des élèves, suite à la séquence, qui ne conduit pas toujours à un choix orthographique normé mais qui peut s'interpréter comme une remise en question des procédures initiales.

Les résultats de la troisième séquence expérimentale traduisent un progrès significatif dans la discrimination des formes verbales homophones en /E/ ainsi que dans l'utilisation pertinente de procédures morphosyntaxiques. Ces résultats ont été discutés dans un autre article (Geoffre, 2014).

\section{Conclusion et perspectives}

Dans le cadre d'une réflexion sur la didactique du français et ses spécificités, notamment en lien avec l'autonymie de la langue, nous avons recherché une approche interdisciplinaire susceptible de nous permettre de nous appuyer sur des stratégies de déstabilisation des représentations issues de la didactique des disciplines scientifiques pour aborder la langue comme un objet d'étude mis à distance et faire évoluer le système orthographique de l'élève. Plus précisément, nous présentons un protocole de mise en œuvre d'un dispositif didactique susceptible de faire progresser l'élève vers une approche morphosyntaxique de l'étude de la langue et à même de développer sa capacité métalinguistique : la phrase dictée/donnée. Ce protocole est une transposition à l'étude de la langue de celui proposé par des didacticiens des sciences.

Ce dispositif a été mis en œuvre lors de deux séquences expérimentales intégrées à notre suivi longitudinal d'une classe de cycle 3 pendant trois années. Les quelques résultats discutés ici montrent une tendance du dispositif à favoriser la compétence réflexive des élèves, la maitrise du métalangage et le traitement des accords dans la chaine syntagmatique. Afin de vérifier la portée de ces résultats, l'étude devrait maintenant s'orienter vers une séquence expérimentale menée dans deux classes différentes : une classe mettant en œuvre la phrase dictée/donnée et une classe témoin proposant une séquence traditionnelle sur la même notion.

\section{BIBLIOGRAPHIE}

Aster (1985), Procédures d'apprentissage en sciences expérimentales, Collection Rapports de recherches, $n^{\circ} 3$, INRP.

Brousseau, G. (1989), « Utilité et intérêt de la didactique pour un professeur de collège », Petit $x$, 21, IREM de Grenoble.

Chevallard, Y. (1991 [1985]), La transposition didactique : du savoir savant au savoir enseigné, Grenoble : La Pensée sauvage.

Chartrand, S. (1995), « Enseigner la grammaire autrement : animer une démarche active de découverte ", Québec français, 99, p. 32-34. 
Cogis, D. (2005), Pour enseigner et apprendre l'orthographe, Paris : Delagrave éditeur.

Dabène, M. (1987), L'adulte et l'écriture, Contribution à une didactique de l'écrit en langue maternelle, Bruxelles : De Boeck-Wesmael.

Garcia-Deband, C. (1990), « Didactique du français et didactiques des disciplines scientifiques : convergences et spécificités ", Actes du colloque de Cerisy : Didactique et pédagogie du français, recherches actuelles, Metz, pp. 41-73.

Geoffre, T. (2014), « Procédures cognitives et formes verbales en /E/ », in Gomila, C., Ulma, D. (dir.), Le verbe en toute complexité - Acquisition, transversalité et apprentissage, Paris : L'harmattan, pp. 192-210.

Geoffre, T. \& Brissaud, C. (2012), « Orthographe grammaticale au cycle 3 : du morphosémantique au morphosyntaxique », in Dumortier J.-L., Van Beveren J. et Vrydaghs D. (dir.), Curriculum et progression en français, Actes du 11e colloque de l'AiRDF (Liège, 26-28 août 2010), Diptyque, 23, Presses Universitaires de Namur.

Gombert, J. E. (1996), « Activités métalinguistiques et acquisition d'une langue », Acquisition et interaction en langue étrangère, 8/1996, Online since 05 December 2011, connection on 26 September 2012. URL : http://aile.revues.org/1224

Guyon, O. (1997), « Acquisition de l"orthographe du CE1 à la $5^{\mathrm{e}}$ : les morphogrammes grammaticaux s et nt ", La linguistique: revue de la Société Internationale de Linguistique Fonctionelle, 33-1, pp. 23-40.

Halté, J.F. (1992), La didactique du français, Paris : PUF.

Sarremajane, P. (2001), « La fabrication des savoirs scolaires », Penser l'éducation, 10, pp. 117-141.

\section{RÉSUMÉS}

Notre réflexion porte sur les procédures cognitives mises en jeu dans l'apprentissage de l'orthographe grammaticale et sur la recherche de dispositifs didactiques permettant de faire progresser l'élève vers une approche morphosyntaxique de l'étude de la langue. On se place dans une optique interdisciplinaire et on propose d'observer des convergences possibles entre didactique de l'orthographe et didactique des sciences. Si l'on considère l'hypothèse que les élèves d'école primaire développent, à partir de leurs apprentissages et savoirs, des procédures cognitives formant un système de représentations et d'outils sur la langue, alors, par analogie, ce système a des limites et ces limites doivent être confrontées à la réalité de "l'expérience ». On cherchera à montrer que deux dispositifs inspirés de protocoles issus de la didactique des sciences peuvent favoriser l'entrée dans une description métalinguistique de la langue.

We report a study concerning the cognitive procedures involved in the learning of grammar and spelling and the search for didactic devices allowing the pupil to progress to a morphosyntactic approach of the study of language. We choose an interdisciplinary approach and we suggest observing possible convergences between didactic of the spelling and didactic of the sciences. If we consider the hypothesis that the pupils of primary school develop, from their learnings and knowledges, cognitive procedures building a system of representations and tools on the language, then, by analogy, this system has limits and these limits must be confronted with the reality of the experiment. We present two devices inspired by protocols stemming from the didactic of the sciences and focus on one that may favor study of the language and control of grammatical agreements. 
INDEX

Keywords : didactic of French, didactic of sciences, cognitive procedures, didactic devices, spelling, grammar

Mots-clés : didactique du français, didactique des sciences, procédures cognitives, dispositifs didactiques, orthographe, grammaire

\section{AUTEUR}

\section{THIERRY GEOFFRE}

Université Grenoble-Alpes LIDILEM

thierry.geoffre@orange.fr 